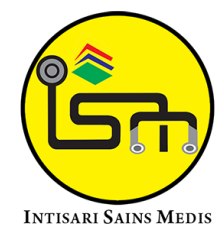

Published by Intisari Sains Medis

\section{Is a high concentration of high-density lipoprotein cholesterol still relevant?}

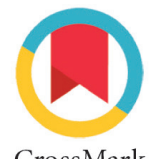

CrossMark

\author{
Hanselim*
}

\section{ABSTRACT}

The importance of cholesterol management, particularly high-density lipoprotein (HDL) cholesterol, has always been believed to be essential in preventing and managing cardiovascular disease (CVD). While HDL has long been accepted as the 'good' cholesterol, various studies have started to challenge this paradigm. Most of these studies found that HDL cholesterol concentrations did not linearly translate into a reduction in CVD risk. There is a suspicion of dysfunctional HDL particles leading to the loss of HDL's cardioprotective function. Some of these dysfunctions were caused by changes in the structure of HDL particles due to an underlying disease or genetic mutations, causing defects in the

reverse cholesterol transport (RCT) mechanism and vascular inflammation inhibition. Therefore, therapies that focus on these two mechanisms are expected to be one of the new cornerstones in reducing CVD. Simultaneously, cholesterol efflux capacity (CEC) assay could become the new therapeutic target as it has an inverse correlation with CVD risk regardless of HDL cholesterol concentration, increasing the accuracy in stratifying CVD risk in patients. However, standardization and a more thorough study on CEC assay and various subcellular cholesterol metabolism should be conducted before stepping further.
*Correspon
Hanselim;

Rumah Sakit Umum Daerah Abdoel Wahab Sjahranie, Samarinda, Kalimantan Timur, Indonesia; hanselim_lim@hotmail.com

Received: 2021-05-04

Accepted: 2021-08-19

Published: 2021-08-31
Keywords: Cardiovascular Disease, Cholesterol Efflux Capacity, Cholesterol Screening, HDL Dysfunction, HighDensity Lipoprotein.

Cite This Article: Hanselim. 2021. Is a high concentration of high-density lipoprotein cholesterol still relevant?. Intisari Sains Medis 12(2): 660-665. D0I: 10.15562/ism.v12i2.1036

\section{INTRODUCTION}

Cardiovascular disease (CVD) is the world's leading cause of death, which is estimated to cause 17.8 million deaths in 2017 , representing $31 \%$ of total deaths in the world. Not only in developed countries, but this estimate also tends to increase in developing countries such as ASEAN, slowly causing the pattern of mortality from communicable to noncommunicable diseases. ${ }^{1}$ Given that most risk factors for CVD can be modified (diet, obesity, activity, alcohol consumption, high cholesterol), preventive efforts should be sought first, both in screening and management. ${ }^{2}$ In addition, several screening instruments that have been used extensively to determine the risk of CVD in a person such as SCORE (Systematic Coronary Risk Evaluation), PCE (ASCVD Risk Estimator), RRS (Reynolds Risk Score), QRISK / JB13 and Framingham CVD Risk Score uses a component of HDL cholesterol concentration in its analysis. ${ }^{2-4}$
Up until now, high-density lipoprotein (HDL) cholesterol has long been labelled as "good" cholesterol in the eye of both health practitioners and the general public. This leads to people thinking that higher HDL cholesterol concentration equals better health. The majority of guidelines focus solely on lowering lowdensity lipoprotein (LDL) cholesterol concentrations. $^{2-4}$ For example, the 2019 European Society of Cardiology/ European Atherosclerosis Society (ESC/ EAS) dyslipidemia guidelines recommend lowering LDL concentrations to at least $50 \%$ of baseline and target LDL cholesterol to be $<55 \mathrm{mg} / \mathrm{dL}$ for patients at very high risk for CVD. These targets vary depending on the stratification of the risk level of each patient. However, there has been no specific target for optimal HDL cholesterol and triglyceride concentrations, although so far, an increase in HDL cholesterol concentrations is thought to reduce the incidence of atherosclerosis. ${ }^{3}$ This knowledge departs from preliminary research, which consistently shows a linear relationship between HDL cholesterol levels with the reduction of CVD. ${ }^{5}$

However, several recent studies have shown the opposite correlation. HDL cholesterol concentration that is too high will cause dire implications to the cardiovascular (CV) system. $^{6-9}$ These findings certainly raise critical questions about the role of HDL cholesterol as a cardio-protector, which will have implications for the medical community, especially in how healthcare providers assess and manage their patients, particularly those who already have a CVD. Furthermore, this phenomenon will also urge us to reconsider the current screening instruments, given that it is true. This paper aims to present the latest knowledge and developments regarding the association of HDL concentrations with CVD risk and its implications for the future. 


\section{CORRELATION OF HDL CHOLESTEROL WITH CVD}

Cholesterol is a lipophilic molecule that has various vital roles in the physiology of the human body. Some of these include precursors for the synthesis of vitamin D and steroid hormones as well as absorption of vitamins in the digestive tract. Due to their lipophilic nature, cholesterol particles require suitable particles to be transported in the blood, namely LDL and HDL. In general, LDL and HDL are particles that have the most prominent role in cholesterol transport. LDL plays a role in transporting cholesterol to peripheral tissues, whereas HDL does the opposite transporting excess cholesterol back to the liver for excretion. This vital mechanism is called reverse cholesterol transport (RCT). ${ }^{10}$ Moreover, HDL in healthy individuals has several protective effects for the vascular system, specifically endothelial cells. HDL stimulates the release of nitric oxide (NO) from endothelial cells, suppresses molecular adhesion, reduce thrombotic activity, repair endothelial cells, and reduces the level of endothelial cell apoptosis. ${ }^{11}$ The most commonly used cholesterol test in the clinical setting is the plasma concentration of HDL cholesterol which is thought to reflect the cholesterol level being transported by HDL particles. Therefore, HDL cholesterol is often referred to as "good" cholesterol, where the higher the concentration, the lower the risk of atherosclerosis.

Nevertheless, Madsen et al. showed a contradictory relationship to our current knowledge concerning the relationship between HDL cholesterol concentration and CVD risk. The study involved 52,268 men and 64,240 women from two cohorts (Copenhagen City Heart Study and Copenhagen General Population Study) and discovered that the association between HDL cholesterol concentration and mortality from all causes had a ' $U$ ' shape curve for both sexes. Hence, suggesting an increased mortality rate in patients with low and very high HDL concentrations. Similar results were also seen in the CANHEART (Cardiovascular Health in Ambulatory Care Research Team) study, an observational cohort involving 631,762 individuals without prior CVD or severe comorbidities. It was observed that individuals with low ( $<50 \mathrm{mg} / \mathrm{dL}$ for women and $<40 \mathrm{mg} /$ $\mathrm{dL}$ for men) and very high (> $80-90 \mathrm{mg}$ ( dL) HDL cholesterol had a higher risk of mortality than individuals with moderate HDL cholesterol concentrations (50 - 79 $\mathrm{mg} / \mathrm{dL}$ ). However, what differentiates this from the previous study is that the mortality rate in individuals with very high HDL cholesterol concentrations is not correlated with CVD mortality. ${ }^{6}$ EPOCH-JAPAN reported a similar finding (2018), where individuals with very high HDL cholesterol (> $90 \mathrm{mg} / \mathrm{dL}$ ) did not experience increased mortality due to CVD. On the other hand, there was an increase in mortality due to $\mathrm{CV}$ atherosclerosis (HR, 2.37; 95\% CI, 1.374.09), chronic heart disease (HR, 2.46, 95\% CI, 1.13 - 5.32), and ischemic stroke (HR, 2.30, 95\% CI, $1.06-4.98){ }^{8}$

Doubts regarding $\mathrm{HDL}$ cholesterol's role as "good" cholesterol are heightened by the results of several clinical trials of drugs aimed at increasing HDL cholesterol. Several studies have tried using cholesteryl ester transfer protein (CETP) inhibitor / anti-CETP. CETP facilitates cholesterol transport from HDL particles to particles containing apolipoprotein B (apo B), such as LDL. ${ }^{12,13}$ Therefore, in theory, inhibition of CETP would increase HDL cholesterol concentration while lowering LDL cholesterol concentrations, which will reduce the risk of atherosclerosis. Thus, using anti-CETP can help to clarify the effect of HDL cholesterol on the risk of CVD.

The first clinical study was ILLUMINATE, a randomized, doubleblind study involving 15,067 patients at high risk of CVD. In this study, patients were divided into the torcetrapib plus atorvastatin group and atorvastatin group. There was an increase in HDL cholesterol concentration up to $72.1 \%$ and a decrease in LDL cholesterol by $24.9 \%$ in the torcetrapib group compared to the placebo group $(\mathrm{P}<0.001)$. Unfortunately, the study was terminated prematurely because of increased morbidity and mortality (95\% CI, 1.14 - 2.19; $\mathrm{P}=0.006$ ), which was thought to result from missed therapeutic targets. ${ }^{14}$ Further investigations showed that torcetrapib caused increased blood pressure and aldosterone production. ${ }^{15}$
The subsequent study is dalOUTCOMES, which was published in 2012. This randomized, double-blind clinical trial involved 15,871 patients who had recently developed the acute coronary syndrome. Participants were divided into two groups, the dalcetrapib (anti-CETP) group and the placebo group. The study was terminated due to an insignificant outcome after 31 months of observation. Although there was an increase in HDL cholesterol (31-40\%) in the dalcetrapib group compared to the placebo group, the primary outcome and total mortality in the study did not produce any significant differences (HR, 1.04; 95\% CI, 0.93-1.16; $\mathrm{P}=0.52$ ). This study's failure is presumed to be due to the insignificant increase in HDL cholesterol; therefore, a more potent anti-CETP is needed. ${ }^{16}$

The third study was ACCELARATE (2017), a randomized, double-blind clinical trial involving 12,092 patients who had experienced CVD 30-365 days earlier. Similar to previous studies, patients will be divided into the evacetrapib (antiCETP) and placebo groups. There was a significant increase in HDL cholesterol concentrations in the evacetrapib group compared to the placebo group (difference of 131.6\%; 95\% CI, 130.0 - 133.1; P <0.001). In addition, there was also a decrease in LDL cholesterol concentrations in the evacetrapib group compared to the placebo group (difference -37.1\%; 95\% CI,-38 - 36.1; P <0.001). However, interim analysis results of the available data in 2015 (82\% final data estimate) did not show any significant difference in key outcomes between the two groups (HR, 1.03; 95\% CI, 0.93 - 1.15; $\mathrm{P}=0.58$ ). On this basis, the study was agreed to be terminated early because of the low efficacy. One plausible hypothesis for the low efficacy was the dysfunction of HDL particles in the study patients, which caused a disturbance in HDL's anti-atherogenic effect. ${ }^{11}$

The most recent study of the use of CETP inhibitors is REVEAL (2017). The randomized, double-blind clinical trial involved 30,449 patients, which is twice as large as previous studies. Patients were divided into the anacetrapib plus statin group and statin plus placebo group. Observation for 4.1 years showed that there was a significant reduction in the 
incidence of coronary disorders (combined coronary death, myocardial infarction, and coronary revascularization) in the anacetrapib group versus the placebo group (1640 of 15,225 patients [10.8\%] vs 1803 of 15,224 patients [11.8\%]; rate ratio, $0.91 ; 95 \%$ CI, $0.85-0.97 ; \mathrm{P}=0.004)$. This difference was even more apparent when assessed in terms of morbidity, risk of myocardial infarction (rate ratio, $0.87 ; 95 \%$ CI, $0.78-0.96 ; \mathrm{P}=0.007)$ and coronary revascularization (rate ratio, $0.90 ; 95 \% \mathrm{CI}$, $0.83-0.97 ; \mathrm{P}=0.01$ ). However, in terms of the death rate from $\mathrm{CV}$ events $(3.4 \%$ in the anacetrapib group vs. $3.7 \%$ in the placebo group, $\mathrm{P}=0.17)$, death other than $\mathrm{CV}$ events $(4.0 \%$ vs. $3.9 \%, P=0.77)$, or death from all events $(7.4 \%$ vs. $7.6 \%, \mathrm{P}=0.46)$ were not seen to be significantly different. Although there was a significant increase in HDL cholesterol in the anacetrapib group (104\% / $43 \mathrm{mg} / \mathrm{dL}$ ) compared to the placebo group, the relative reduction in risk of coronary death or myocardial infarction was only $11 \%$, similar to the results of a similar previous study (10\%). ${ }^{17}$ Moreover, based on previous studies, this risk reduction mechanism is speculated to be the impact of reducing non-HDL cholesterol rather than the increasing HDL cholesterol. ${ }^{18}$ Thus, It can be concluded that an increase in HDL concentration does not have an impact on reducing mortality due to coronary disorders but on reducing the risk of coronary disorders.

Broadly speaking, the failure of these four studies poses big questions to the role of HDL as a cardioprotector. Perhaps it suggests that the high concentration of HDL cholesterol does not necessarily imply a reduction in CVD incidence but solely as a marker of CVD or a person's wellbeing. ${ }^{6}$ Furthermore, this may indicate a dysfunction of HDL cholesterol that causes disturbance in HDL cholesterol's cardioprotective mechanism, or even worse, to become dangerous. ${ }^{19,20}$

\section{GENETIC MUTATION IN HDL}

Apart from various external factors that cause an increase in HDL cholesterol, it might be wiser to start investigating from the internal factors. Up to now, at least three HDL metabolism components are considered to play a pivotal role in this phenomenon, mainly the scavenger receptor class BI (SR-BI), CETP, and the endothelial lipase gene (LIPG). The discovery of the SR-BI encoded by the SCARB1 gene has become one of the pioneers of fundamental knowledge on HDL metabolism. SR-B1 bridges the selective transfer of HDL cholesteryl ester (HDL-CE) to enter liver cells to metabolize the cholesterol in HDL particles. ${ }^{21}$ Further studies have shown that overexpression of SR-BI reduces the risk of atherosclerosis despite low HDL cholesterol concentrations; moreover, deletion of the gene increases the risk of atherosclerosis despite high HDL cholesterol concentrations. One possible explanation is the flow of cholesterol from macrophages through the RCT mechanism, where overexpression of SRB1 will increase macrophages' RCT and vice versa. ${ }^{22,23}$

$\mathrm{P} 376 \mathrm{~L}$ is a variant of the SCARB1 gene that mutates and results in the loss of the main function of SR-BI in transporting HDL cholesterol selectively. The implication of this mutation is an increased risk of coronary heart disease for heterozygous carriers of the $\mathrm{P} 376 \mathrm{~L}$ variant. (OR, 1.79; $\mathrm{P}=0.018)$. Moreover, there is a shift in the composition of HDL to large HDL particles, while it is known that large HDL particles have weaker antioxidant and anti-inflammatory properties than small HDL particles. ${ }^{23,24}$

The second component is the LIPG gene which encodes endothelial lipase. Endothelial lipase acts as a negative regulator of HDL cholesterol concentration, which means an increase in this enzyme will lower HDL cholesterol and vice versa. A Mendelian study showed that individuals with the LIPG Asn396Ser allele polymorphism had higher concentrations of HDL cholesterol without a reduced risk of myocardial infarction (OR: 0.99, 95\% CI, 0.88 - 1.11, P $=0.85) \cdot{ }^{25} \mathrm{~A}$ similar outcome was seen in a recent meta-analysis by Zhao et al. (2020), who found a higher suspicion of coronary artery disease in the carrier of $584 \mathrm{C} / \mathrm{T}$, a mutation of the LIPG gene. ${ }^{26}$

CETP rs708272 (C>T) and rs1800775 (C>A), are CETP polymorphisms which are crucial components in HDL metabolism. Individuals with these polymorphisms have a higher risk of myocardial infarction.
However, this phenomenon is only found in Caucasians whose mechanisms are still not fully understood. ${ }^{27}$ Despite various failures in CETP inhibitors' clinical trials, it is possible that these polymorphisms can be a marker for the early diagnosis of myocardial infarction. Nonetheless, studies with larger sample sizes and involving multiple ethnicities are needed.

In conclusion, there is strong evidence that these genetic variants have implications for HDL dysfunction. Nevertheless, some of these variants are classified as rare, and their frequency is low. Therefore, it might be too immature to attribute this to the high mortality rate in previous studies. Furthermore, some of these studies are limited by their small sample sizes, less variety of ethnicity, and numerous unstudied HDL genes, demanding a further investigation to strengthen the concept.

\section{STRUCTURAL CHANGES IN HDL}

This new development demands a change in the focus of investigation from merely HDL cholesterol concentration to the function of HDL particles. These functional changes are mainly observed in patients with cardiovascular, kidney, and metabolic diseases. ${ }^{28-31}$ To date, there are still numerous HDL related molecules that are not fully understood regarding their role and its implication if it is dysfunction. However, in general, there are changes in HDL's protein and lipid structure due to various mechanisms such as oxidation or glycation that eventually bring forth the same consequence, a decrease in the cardioprotective function of HDL.

As mentioned previously, the majority of diseases cause a reduction in the amount of sphingosine 1-phosphate (S1P) in blood plasma. ${ }^{32} \mathrm{~S} 1 \mathrm{P}$ is a lipid mediator that activates the sphingosine 1-phosphate 1 (S1P1) receptor located on endothelial cells. S1P1 activation will later provide a cardioprotective function through suppression of vascular inflammation and atherosclerosis. ${ }^{33}$ This mechanism may be the basis for the study outcomes by Yuji et al. (2019), who found endothelial dysfunction in male patients with very high HDL cholesterol concentrations $(\geq 80$ $\mathrm{mg} / \mathrm{dL}) .^{34}$ This dysfunction is indicated by a decrease in flow-mediated vasodilation 
(FMD), which is known to have a strong correlation with CVD risk factors. Besides, S1P needs to bond with Apolipoprotein $\mathrm{M}$ (ApoM) on HDL in order to work. In patients with metabolic disorders such as uremia, the ApoM concentration is reduced and results in the inactivation of S1P. ${ }^{35}$

Another HDL particle structure that is often become the target of modification is Apolipoprotein AI (ApoAI). ApoAI is the most abundant apolipoprotein in HDL and plays an essential role in RCT and cellular cholesterol homeostasis. ${ }^{36}$ Modifications to apoAI can occur due to glycation and oxidation processes. The oxidation process is mediated by myeloperoxidase (MPO), a component of the human immune system that functions to kill microbes through the formation of reactive oxidants and free radicals. When MPO circulates in the blood vessels, it can bind to apoAI in HDL, which cause damage and resulting in apoAI dysfunction. ${ }^{37}$ Likewise, this mechanism also happened in glycation. Glycation is the process of adding sugars covalent to proteins or lipids. Aside from apoAI dysfunction, glycation also reduces the S1P concentration in HDL, leading to increased cardiomyocyte cell death due to ineffective activation of the intracellular survival pathway. ${ }^{29,37}$

A study by Vaisar et al. (2015) shows that acute inflammation affects the efflux capacity of HDL cholesterol regardless of HDL cholesterol concentration. The proteomic analysis demonstrated that this cholesterol efflux capacity is closely related to serum amyloid A (SAA) 1 and SAA2 in HDL particles. Acute inflammation will cause changes in the HDL structure mediated by SAA, resulting in a decrease in HDL cholesterol's efflux capacity. ${ }^{38}$ This finding is supported by Tölle et al. (2012), in which patients with chronic inflammation showed an increase in SAA concentration, which resulted in the stimulation of monocyte chemoattractant protein-1 (MCP-1) production. MCP1 is a pro-inflammatory molecule that contributes to the initiation and progression of atherosclerosis. ${ }^{31}$

Based on the various mechanisms described above, it can be concluded that HDL's reduced cardioprotective function occurs due to decreased RCTs and failure of inhibition of vascular inflammation. Therapy that focuses on both of these may lower CVD risk and preferably be sought rather than merely focusing on $\mathrm{HDL}$ cholesterol concentrations. Although there are still various other factors that contribute indirectly, and until further research, a definite conclusion still cannot be drawn.

\section{HDL AS A THERAPY TARGET}

In the future, the examination of $\mathrm{HDL}$ cholesterol concentration may become less relevant in determining an individual's CV risk. A more sensitive and specific test is needed, and by far, the cholesterol efflux capacity (CEC) test is probably one of the most likely candidates. The cholesterol efflux capacity test is used to check the capacity of HDL particles to remove cholesterol from cells in vitro without modifying or eliminating the subpopulation of HDL to simulate the body's HDL metabolism closely. ${ }^{39}$ The CEC test principally requires two components: donor cells that will excrete the labelled cholesterol and cholesterol acceptor cells (serum HDL from the patient).

A meta-analysis showed that a high CEC test result was associated with a reduced $\mathrm{CV}$ risk than the lowest CEC (RR, 0.56\%; 95\% CI, 0.37-0.85; I $\mathrm{I}^{2}$, $89 \%)$. Furthermore, compared with the lowest CEC, the prevalence of CVD was significantly reduced in the participants with the highest CEC (OR, 0.30; 95\% CI, $\left.0.17-0.51 ; \mathrm{I}^{2}=71 \%\right)$. Nevertheless, there was no significant difference in the highest CEC and lowest CEC groups regarding allcause mortality. This finding indicates that CEC is more closely associated with $\mathrm{CV}$ risk and not with other risks. It should be noted that the type of CVD in this study is not specific. Thus a larger clinical trial is needed to validate this concept. Moreover, there is no primary benchmark for the CEC test, so differences in donor cell type (J774 or THP-1) and labelled cholesterol (fluorescent or radiolabel) can produce varying results. ${ }^{40}$

A more recent study by Ritsch et al. (2020) showed that cholesterol efflux could predict mortality due to CVD regardless of HDL cholesterol level. Moreover, CEC is also associated with various HDL parameters (varying HDL size and HDL composition) and various inflammatory markers (CRP, Fibrinogen, Il-6 and SAA). A high level of CEC indicates a low level of inflammation and vice versa. In other words, inflammatory conditions cause HDL dysfunction, which translates into decreased RCTs. These findings support the study of Vaiser et al. on the concept of inflammation and HDL dysfunction. ${ }^{38}$ However, it should be noted that this study was conducted in vitro, as was the case with other CEC studies, which means that there are still some subcellular mechanisms of cholesterol metabolism in the body that were not involved. ${ }^{41}$

CEC is an essential key in the RCT system to the liver. Hence, adding a CEC assay to the traditional risk factors is expected to increase the accuracy in stratifying CVD risk. ${ }^{42}$ However, before the CEC assay can be widely applied in the clinical setting, it is necessary to standardize the CEC assay so that each examination result produces similar accurate results. Moreover, the CEC assay is principally done in vitro; therefore, it requires a comprehensive understanding of the various subcellular mechanisms of cholesterol metabolism in the body, which must then be applied to the analysis. This is vital so that the CEC assay can provide an accurate representation of our body HDL function.

\section{CONCLUSION}

The current paradigm of HDL cholesterol as the "good cholesterol" might need to be reconsidered. An increasing number of studies challenged this paradigm and indicate that the relationship between HDL cholesterol and CVD risk factors is " $U$ " shaped. This relationship curve explains that very high HDL concentrations also increased mortality rate, which then questions the role of HDL cholesterol in stratifying the risk of CVD. Moreover, studies on drugs aimed to increase HDL cholesterol concentration failed to reduce mortality raise a suspicion that HDL particles might be dysfunctional, leading to the loss of HDL's cardioprotective function caused by changes in the structure of HDL particles due to an underlying disease or genetic mutations. Additionally, the CEC assay measure of HDL RCT function showed 
an inverse correlation with CVD risk and the level of inflammation regardless of HDL cholesterol concentration. Therefore, the CEC assay could improve the accuracy of CVD risk stratification. Nevertheless, standardization and a more thorough study on CEC assay and various subcellular cholesterol metabolism should be conducted before stepping further.

\section{ACKNOWLEDGEMENTS}

The author would like to thank dr. Denio Adrianus Ridjab, Sp. JP (K) as the mentor who supports this review article.

\section{CONFLICT OF INTEREST}

The author reports no conflicts of interest in this work.

\section{FUNDING}

No third party or funding agency involved in this work.

\section{REFERENCES}

1. WHO CVD Risk Chart Working Group. World Health Organization cardiovascular disease risk charts: revised models to estimate risk in 21 global regions. Lancet Glob Health. 2019;7(10):e1332-45.

2. Arnett DK, Blumenthal RS, Albert MA, Buroker $\mathrm{AB}$, Goldberger ZD, Hahn EJ, et al. 2019 ACC/AHA Guideline on the Primary Prevention of Cardiovascular Disease: A Report of the American College of Cardiology/ American Heart Association Task Force on Clinical Practice Guidelines. Circulation. 2019;140(11):e596-646. Available from: https://www.ahajournals.org/doi/10.1161/ CIR.0000000000000678

3. Mach F, Baigent C, Catapano AL, Koskinas KC, Casula M, Badimon L, et al. 2019 ESC/ EAS Guidelines for the management of dyslipidaemias: lipid modification to reduce cardiovascular risk: The Task Force for the management of dyslipidaemias of the European Society of Cardiology (ESC) and European Atherosclerosis Society (EAS). Eur Heart J. 2020;41(1):111-88. Available from: https://doi. org/10.1093/eurheartj/ehz455

4. Huang Y-C, Lin C-Y, Lan C-C, Wu Y-K, Lim C-S, Huang $\mathrm{C}-\mathrm{Y}$, et al. Comparison of cardiovascular comorbidities and CPAP use in patients with positional and non-positional mild obstructive sleep apnea. BMC Pulm Med. 2014;14:153-153. Available from: https://pubmed.ncbi.nlm.nih. gov/25257571

5. Wilson PW, Abbott RD, Castelli WP. High density lipoprotein cholesterol and mortality. The Framingham Heart Study. Arterioscler Off J Am Heart Assoc Inc. 1988;8(6):737-41. Available from: http://dx.doi.org/10.1161/01. atv.8.6.737
6. Ko DT, Alter DA, Guo H, Koh M, Lau G, Austin PC, et al. High-Density Lipoprotein Cholesterol and Cause-Specific Mortality in Individuals Without Previous Cardiovascular Conditions. J Am Coll Cardiol. 2016;68(19):2073-83. Available from: http:// dx.doi.org/10.1016/j.jacc.2016.08.038

7. Nordestgaard BG, Madsen CM, Varbo A. Extreme high high-density lipoprotein cholesterol is paradoxically associated with high mortality in men and women: Two prospective cohort studies. Atherosclerosis. 2017;263:e89. Available from: http://dx.doi.org/10.1016/j. atherosclerosis.2017.06.291

8. Hirata A, Sugiyama D, Watanabe M, Tamakoshi A, Iso $\mathrm{H}$, Kotani $\mathrm{K}$, et al. Association of extremely high levels of high-density lipoprotein cholesterol with cardiovascular mortality in a pooled analysis of 9 cohort studies including 43,407 individuals: The EPOCH-JAPAN study. J Clin Lipidol. 2018;12(3):674-684.e5. Available from: http://dx.doi.org/10.1016/j. jacl.2018.01.014

9. Li X, Guan B, Wang Y, Tse G, Zou F, Khalid BW, et al. Association between high-density lipoprotein cholesterol and all-cause mortality in the general population of northern China. Sci Rep. 2019;9(1):14426-14426. Available from: https://pubmed.ncbi.nlm.nih.gov/31594968

10. Wang HH, Garruti G, Liu M, Portincasa P, Wang DQ-H. Cholesterol and Lipoprotein Metabolism and Atherosclerosis: Recent Advances in Reverse Cholesterol Transport. Ann Hepatol. 2017;16:S27-42. Available from: http://dx.doi.org/10.5604/01.3001.0010.5495

11. Lüscher TF, Landmesser U, von Eckardstein A, Fogelman AM. High-Density Lipoprotein. Circ Res. 2014;114(1):171-82. Available from: http:// dx.doi.org/10.1161/circresaha.114.300935

12. Davidson MH. Update on CETP inhibition. J Clin Lipidol. 2010;4(5):394-8. Available from: http://dx.doi.org/10.1016/j.jacl.2010.08.003

13. Johns DG, Duffy J, Fisher T, Hubbard BK, Forrest MJ. On- and Off-Target Pharmacology of Torcetrapib. Drugs. 2012;72(4):491507. Available from: http://dx.doi. org/10.2165/11599310-000000000-00000

14. Barter PJ, Caulfield M, Eriksson M, Grundy SM, Kastelein JJP, Komajda M, et al. Effects of Torcetrapib in Patients at High Risk for Coronary Events. N Engl J Med. 2007;357(21):2109-22. Available from: http:// dx.doi.org/10.1056/nejmoa0706628

15. Clerc RG, Stauffer A, Weibel F, Hainaut E, Perez A, Hoflack J-C, et al. Mechanisms underlying off-target effects of the cholesteryl ester transfer protein inhibitor torcetrapib involve L-type calcium channels. J Hypertens. 2010;28(8):1676-86. Available from: http:// dx.doi.org/10.1097/hjh.0b013e32833b1f8e

16. Schwartz GG, Olsson AG, Abt M, Ballantyne CM, Barter PJ, Brumm J, et al. Effects of Dalcetrapib in Patients with a Recent Acute Coronary Syndrome. N Engl J Med. 2012;367(22):2089-99. Available from: http:// dx.doi.org/10.1056/nejmoa1206797

17. HPS3/TIMI55-REVEAL Collaborative Group, Bowman L, Hopewell JC, Chen F, Wallendszus $\mathrm{K}$, Stevens W, et al. Effects of Anacetrapib in
Patients with Atherosclerotic Vascular Disease. N Engl J Med. 2017;377(13):1217-27.

18. Cholesterol Treatment Trialists' (CTT) Collaboration, Baigent C, Blackwell L, Emberson J, Holland LE, Reith C, et al. Efficacy and safety of more intensive lowering of LDL cholesterol: a meta-analysis of data from 170,000 participants in 26 randomized trials. Lancet Lond Engl. 2010/11/08 ed. 2010;376(9753):1670-81. Available from: https://pubmed.ncbi.nlm.nih. gov/21067804

19. Barter PJ, Rye K-A. HDL cholesterol concentration or HDL function: which matters? Eur Heart J. 2017;38(32):2487-9. Available from: http://dx.doi.org/10.1093/eurheartj/ ehx 274

20. Filippatos TD, Elisaf MS. High density lipoprotein and cardiovascular diseases. World J Cardiol. 2013;5(7):210-4. Available from: https://pubmed.ncbi.nlm.nih.gov/23888190

21. Acton S, Rigotti A, Landschulz KT, Xu S, Hobbs $\mathrm{HH}$, Krieger M. Identification of Scavenger Receptor SR-BI as a High Density Lipoprotein Receptor. Science. 1996;271(5248):518-20. Available from: http://dx.doi.org/10.1126/ science.271.5248.518

22. Samadi S, Farjami Z, Hosseini ZS, Ferns GA, Mohammadpour A hooshang, Tayefi M, et al. Rare P376L variant in the SR-BI gene associates with HDL dysfunction and risk of cardiovascular disease. Clin Biochem. 2019;73:44-9. Available from: http://dx.doi. org/10.1016/j.clinbiochem.2019.06.014

23. Zanoni P, Khetarpal SA, Larach DB, HancockCerutti WF, Millar JS, Cuchel M, et al. Rare variant in scavenger receptor BI raises HDL cholesterol and increases risk of coronary heart disease. Science. 2016;351(6278):1166-71. Available from: https://pubmed.ncbi.nlm.nih. gov/26965621

24. Silbernagel G, Pagel P, Pfahlert V, Genser B, Scharnagl H, Kleber ME, et al. HighDensity Lipoprotein Subclasses, Coronary Artery Disease, and Cardiovascular Mortality. Clin Chem. 2017;63(12):1886-96. Available from: http://dx.doi.org/10.1373/ clinchem.2017.275636

25. Voight BF, Peloso GM, Orho-Melander M, Frikke-Schmidt R, Barbalic M, Jensen MK, et al. Plasma HDL cholesterol and risk of myocardial infarction: a mendelian randomization study. Lancet Lond Engl. 2012;380(9841):572-80.

26. Zhao H, Hu S, Rong J. Gene polymorphism associated with Angiotensinogen(M235T), Endothelial lipase $(584 \mathrm{C} / \mathrm{T})$ and susceptibility to coronary artery disease: A metaanalysis. 2020; Available from: http://dx.doi. org/10.1101/2020.04.24.059295

27. Wang Q, Zhou S-B, Wang L-J, Lei M-M, Wang Y, Miao C, et al. Seven functional polymorphisms in the CETP gene and myocardial infarction risk: a meta-analysis and meta-regression. PloS One. 2014;9(2):e88118-e88118. Available from: https://pubmed.ncbi.nlm.nih.gov/24533069

28. Riwanto M, Rohrer L, Roschitzki B, Besler C, Mocharla P, Mueller M, et al. Altered Activation of Endothelial Anti- and Proapoptotic Pathways by High-Density Lipoprotein from Patients with Coronary Artery Disease. Circulation. 
2013;127(8):891-904. Available from: http:// dx.doi.org/10.1161/circulationaha.112.108753

29. Brinck JW, Thomas A, Lauer E, Jornayvaz FR, Brulhart-Meynet M-C, Prost J-C, et al. Diabetes Mellitus Is Associated With Reduced High-Density Lipoprotein Sphingosine1-Phosphate Content and Impaired HighDensity Lipoprotein Cardiac Cell Protection. Arterioscler Thromb Vasc Biol. 2016;36(5):81724. Available from: http://dx.doi.org/10.1161/ atvbaha.115.307049

30. Morgantini C, Natali A, Boldrini B, Imaizumi $S$, Navab M, Fogelman AM, et al. Antiinflammatory and antioxidant properties of HDLs are impaired in type 2 diabetes. Diabetes. 2011/08/18 ed. 2011;60(10):2617-23. Available from: https://pubmed.ncbi.nlm.nih. gov/21852676

31. Tölle M, Huang T, Schuchardt M, Jankowski V, Prüfer N, Jankowski J, et al. High-density lipoprotein loses its anti-inflammatory capacity by accumulation of pro-inflammatory-serum amyloid A. Cardiovasc Res. 2012;94(1):154-62. Available from: http://dx.doi.org/10.1093/cvr/ cvs089

32. Levkau B. HDL-S1P: cardiovascular functions, disease-associated alterations, and therapeutic applications. Front Pharmacol. 2015;6:243-243. Available from: https://pubmed.ncbi.nlm.nih. gov/26539121

33. Galvani S, Sanson M, Blaho VA, Swendeman $\mathrm{SL}$, Obinata $\mathrm{H}$, Conger $\mathrm{H}$, et al. HDL-bound sphingosine 1-phosphate acts as a biased agonist for the endothelial cell receptor S1P1 to limit vascular inflammation. Sci Signal. 2015;8(389):ra79-ra79. Available from: https:// pubmed.ncbi.nlm.nih.gov/26268607

34. Takaeko Y, Matsui S, Kajikawa M, Maruhashi T, Kishimoto S, Hashimoto H, et al. Association of extremely high levels of high-density lipoprotein cholesterol with endothelial dysfunction in men. J Clin Lipidol. 2019;13(4):664-672.e1. Available from: http://dx.doi.org/10.1016/j. jacl.2019.06.004

35. Holzer M, Birner-Gruenberger R, Stojakovic T, El-Gamal D, Binder V, Wadsack C, et al. Uremia alters HDL composition and function. J Am Soc Nephrol JASN. 2011/07/29 ed. 2011;22(9):1631-41. Available from: https:// pubmed.ncbi.nlm.nih.gov/21804091

36. Mangaraj M, Nanda R, Panda S. Apolipoprotein A-I: A Molecule of Diverse Function. Indian J Clin Biochem IJCB. 2015/07/16 ed. 2016;31(3):253-9. Available from: https:// pubmed.ncbi.nlm.nih.gov/27382195

37. Fisher EA, Feig JE, Hewing B, Hazen SL, Smith JD. High-density lipoprotein function, dysfunction, and reverse cholesterol transport. Arterioscler Thromb Vasc Biol. 2012;32(12):2813-20. Available from: https:// pubmed.ncbi.nlm.nih.gov/23152494

38. Vaisar T, Tang C, Babenko I, Hutchins P, Wimberger J, Suffredini AF, et al. Inflammatory remodeling of the HDL proteome impairs cholesterol efflux capacity. J Lipid Res. 2015/05/20 ed. 2015;56(8):1519-30.
Available from: https://pubmed.ncbi.nlm.nih. gov/25995210

39. Anastasius M, Kockx M, Jessup W, Sullivan D, Rye K-A, Kritharides L. Cholesterol efflux capacity: An introduction for clinicians. Am Heart J. 2016;180:54-63. Available from: http:// dx.doi.org/10.1016/j.ahj.2016.07.005

40. Qiu C, Zhao X, Zhou Q, Zhang Z. Highdensity lipoprotein cholesterol efflux capacity is inversely associated with cardiovascular risk: a systematic review and meta-analysis. Lipids Health Dis. 2017;16(1):212-212. Available from: https://pubmed.ncbi.nlm.nih.gov/29126414

41. Ritsch A, Duerr A, Kahler P, Hunjadi M, Stojakovic T, Silbernagel G, et al. Cholesterol Efflux Capacity and Cardiovascular Disease: The Ludwigshafen Risk and Cardiovascular Health (LURIC) Study. Biomedicines. 2020;8(11):524. Available from: https://pubmed.ncbi.nlm.nih. gov/33233452

42. Rohatgi A, Khera A, Berry JD, Givens EG, Ayers $\mathrm{CR}$, Wedin KE, et al. HDL cholesterol efflux capacity and incident cardiovascular events. $\mathrm{N}$ Engl J Med. 2014;371(25):2383-93.

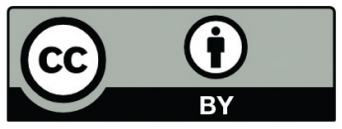

This work is licensed under a Creative Commons Attribution 